\title{
33 Perioperatives Management
}

Wolfgang Schwenk, Yasmin Hosseini-Milbradt

\subsection{Kapitelzusammenfassung}

Bereits vor mehr als 20 Jahren wurden die ersten evidenzbasierten, interprofessionellen und multimodalen Behandlungskonzepte zum perioperativen Management als sogenannte Fast-track-Chirurgie oder ERAS (,enhanced recovery after surgery - beschleunigte Genesung nach Operationen“) für die wichtigsten Eingriffe in der Allgemein- und Viszeralchirurgie formuliert. Unverändert stellt die elektive Kolonresektion das am besten untersuchte Modell für den Erfolg multimodaler Behandlungskonzepte dar. Daher wird das perioperative Management bei dieser Operation im Folgenden auch beispielhaft beschrieben. Obwohl die Bedeutung der einzelnen Elemente des Fast-track noch nicht ausreichend erforscht ist, gelten folgende 6 Maßnahmen als essentielle Kernelemente erfolgreicher multimodaler Behandlungskonzepte:

1. präoperative Patienteninformation und -motivation

2. adäquate Schmerztherapie (durch thorakale Periduralanalgesie bei offenen Operationen, aber nicht bei laparoskopischer Chirurgie)

3. minimalinvasive Operationstechnik

4. Vermeidung perioperativer Hyper- und Hypovolämie

5. Verzicht auf Sonden und Drainagen mit frühzeitiger oraler Ernährung und

6. forcierte, frühe postoperative Mobilisation.

Weitere perioperative Maßnahmen sind: präoperative Optimierung, Behandlung einer präoperativen Anämie, kurze präoperative Nüchternheit, präoperative kohlenhydratreiche Trinklösungen, mechanische Darmvorbereitung, präoperative orale Antibiotikagabe, Prophylaxe von postoperativer Übelkeit und Erbrechen (PONV), Vermeidung langwirksamer Sedativa zur Prämedikation, perioperative Antibiotikaprophylaxe, intraoperative Aufrechterhaltung der Körpertemperatur, standardisierte Narkose, medikamentöse Thromboseprophylaxe, postoperative Infusionstherapie, Verzicht oder frühe Entfernung von Blasenkathetern und Prophylaxe der postoperativen Darmatonie.

Da die Compliance mit den zahlreichen Bausteinen des Fast-track eng mit dem Erfolg der Behandlung assoziiert ist, muss die Umsetzung möglichst aller Maßnahmen exakt koordiniert und überwacht werden. Mit dieser Aufgabe sind sowohl Ärzte als auch Pflegende überfordert, so dass die erfolgreiche Umsetzung im klinischen Alltag an spezielles Personal, sogenannte ERAS-Schwestern oder Fast-track-Assistenten, gebunden ist. 


\subsection{Definition}

Fast-track und ERAS sind synonyme Bezeichnungen für ein multimodales, evidenzbasiertes, interprofessionelles, prozedurenspezifisches und patientenzentriertes perioperatives Behandlungskonzept (da der Begriff „ERAS“ durch ein europaweites Copyright geschützt zu sein scheint, wird im Folgenden ausschließlich der Begriff Fast-track verwendet). Multimodal, weil einzelne Maßnahmen keinen umfassenden Einfluss auf die komplexen pathophysiologischen Vorgänge in der perioperativen Phase haben können. Evidenzbasiert, weil die Wirksamkeit der meisten Einzelmaßnahmen ebenso wie die positiven Effekte des gesamten Behandlungskonzeptes durch die Ergebnisse randomisierter, kontrollierter klinischer Studien mit hohem Empfehlungsgrad belegt worden sind. Interprofessionell, weil eine enge Abstimmung aller Berufsgruppen (Ärzte, Pflegende, Physiotherapie, Sozialdienst, medizinisches Hilfspersonal) erforderlich ist, um das gewünschte Ergebnis zu erzielen. Prozedurenspezifisch, weil zwar die Grundregeln des Behandlungskonzeptes für alle Operationen gelten, aber eine Anpassung an die jeweilige Operation (oder eine Gruppe von Operationen) erforderlich ist. Patientenzentriert, weil nur die aktive Mitarbeit des Patienten den Erfolg der perioperativen Behandlung sichert und diese Mitarbeit nur durch eine intensive Zuwendung zum Patienten erreicht wird.

\subsection{Optimales perioperatives Management}

Im Folgenden werden die Erkenntnisse zu den einzelnen Maßnahmen des perioperativen Managements kurz zusammengefasst. Als Beispiel wird dabei die elektive Kolonresektion gewählt, obwohl auch Patienten mit elektiver hoher anteriorer Rektumresektion und partieller mesorektaler Exzision, Rektopexie (mit und ohne Resektion) oder anderen transabdominellen koloproktologischen Operationen analog behandelt werden können. Fast-track-Behandlungspfade für elektive tiefe anteriore Rektumresektionen mit totaler mesorektaler Exzision und protektivem Stoma, abdominoperineale Rektumexstirpationen oder Proktokolektomien mit protektivem Stoma sind ähnlich, müssen aber den Besonderheiten der genannten Eingriffe (Stoma, perineale Wunde) Rechnung tragen.

\subsubsection{Optimales präoperatives Management}

\section{Kernelemente der optimalen pråoperativen Behandlung}

Präoperative Patienteninformation und -motivation: Eine sorgfältige Patientenaufklärung und psychologische Unterstützung führt zu einer Minderung der perioperativen Ängste und Befürchtungen der Patienten und geht mit einer rascheren Genesung und einem verkürzten postoperativen Krankenhausaufenthalt einher. Da die aktive Mit- 
arbeit der Patienten in der frühen postoperativen Phase entscheidend für den Erfolg multimodaler perioperativer Behandlungskonzepte ist, muss die ausführliche Patientenschulung unbedingt bereits vor der Aufnahme ins Krankenhaus erfolgen.

\section{Weitere Elemente der optimalen pråoperativen Behandlung}

Präoperative Optimierung: Evidenzbasierte Empfehlungen der wissenschaftlichen Fachgesellschaften zur präoperativen Risikoevaluation und Patientenoptimierung liegen vor. Ein Ernährungs-Screening sollte bei allen Patienten vor größeren Operationen erfolgen, obwohl eine Optimierung durch präoperative Alimentation nur bei mangelernährten Hoch-Risiko-Patienten gelungen ist. Nikotin- und Alkoholabstinenz für 4-8 Wochen vor der Operation verbessern die Ergebnisse, sind aber oftmals nur sehr schwer zu realisieren.

Behandlung einer präoperativen Anämie: Eine präoperative Anämie $(\mathrm{Hb}<13 \mathrm{~g} /$ dl beim Mann, <12 g/dl bei nicht-schwangeren Frauen, $<11 \mathrm{~g} / \mathrm{dl}$ bei schwangeren Frauen) verfünffacht die postoperative Sterblichkeit im Vergleich zu Patienten mit normalem präoperativem Hämoglobin-Wert. Gemäß der aktuellen S3-Leitlinie ist eine zweistufige Diagnostik und nachfolgende Therapie der präoperativen Anämie bei Eingriffen mit einem Transfusionsrisiko von $>10 \%$ zwingend indiziert. Elektive Eingriffe sollten bei anämischen Patienten daher um 4-6 Wochen verschoben werden, um den Hb-Wert anzuheben .

Kurze präoperative Nüchternheit: Erwachsene Patienten ohne relevante Magenentleerungsstörungen dürfen bis 6 Stunden vor der Operation feste Nahrung zu sich nehmen und sollten bis 2 Stunden präoperativ klare Flüssigkeiten trinken.

Präoperative kohlenhydratreiche Trinklösungen: Die orale Gabe von 400-800 ml $12 \%$ Maltodextrinhaltiger klarer Trinklösungen am Abend vor der Operation und weiterer 200-400 ml dieser Flüssigkeiten 2Stunden präoperativ kann die katabolische Reaktion des Organismus auf das Operationstrauma dämpfen und den postoperativen Hospitalaufenthalt verkürzen .

Mechanische Darmvorbereitung: Die sogenannte mechanische Darmvorbereitung (,mechanical bowel preparation“ - MBP) wurde unter der Vorstellung eingeführt, dass ein weniger stark stuhlverschmutzter Darm mit niedrigeren Raten Anastomoseninsuffizienzen und Wundheilungsstörungen einhergeht. Initial wurden zur MBP bis zu 101 Elektrolytlösungen via Magensonde verabreicht, später wurden Trinklösungen entwickelt (Polyethylenglykol-Lösung) um die zugeführte Flüssigkeitsmenge zu reduzieren und heute ist die MBP mit geringen Mengen Natriumphosphat-Lösung und begleitendem Trinken von $<1.000 \mathrm{ml}$ möglich. In den initialen Publikationen zum Fast-track wurde die MBP nicht thematisiert. Später zeigten randomisierte, kontrollierte Studien und ihre Metaanalysen keine Vorteile einer MBP vor elektiven Kolonresektionen und die negativen Auswirkungen auf die Herz-Kreislauf-Physiologie wurden stärker beachtet. Heute sollte auf die alleinige MBP vor elektiven Kolonresektionen verzichtet werden, vor tiefen anterioren Rektumresektionen mit protektivem Ileostoma kann die 
MBP dagegen sinnvoll sein. Ob die Kombination der MBP mit einer oralen Antibiotikagabe positive Effekte haben könnte, ist derzeit Gegenstand der Diskussion.

Präoperative orale Antibiotikagabe: Die zusätzliche Gabe oraler Antibiotika am Tag vor einer geplanten kolorektalen Resektion könnte die Inzidenz postoperativer infektiöserer Komplikationen im Operationsgebiet reduzieren.

PONV-Prophylaxe: Postoperative Übelkeit und Erbrechen werden bei bis zu $80 \%$ der Patienten beobachtet, die einen mehr als einstündigen intraabdominellen Eingriff mit Opioidgabe während der Narkose unterlaufen. Daher ist eine PONV-Prophylaxe zumindest bei Risikopatienten heute unumstritten und Patienten mit gastrointestinalen Operationen profitieren von einer einmaligen präoperativen Gabe von $8 \mathrm{mg}$ Dexamethason.

Adäquate Prämedikation: Benzodiazepine sind früher vor elektiven Operationen aufgrund ihrer anxiolytischen Wirkung als Prämedikation verwendet worden. Allerdings können diese Substanzen auch bei Einmalgaben anhaltende psychomotorische und kognitive Störungen mit andauernder Sedierung hervorrufen. Daher sollten diese Substanzen nicht zur Prämedikation verwendet werden. Ob die Prämedikation mit Gabapentin oder nichtsteroidalen Antiphlogistika die perioperative Opioid-Dosis reduzieren kann, ist umstritten.

\subsubsection{Optimales Management im Operationssaal}

\section{Kernelemente der optimalen Behandlung im Operationssaal}

Adäquate Schmerztherapie: Generell sollten Anästhesie- und Analgesietechniken verwendet werden, welche die perioperative Opioid-Dosis senken, so dass vor allem neuroaxiale Lokalanästhetikablockaden (Periduralanalgesie, Spinalanalgesie) und regionalanästhetische Techniken (Transversus Abdominis Plane Blockade-TAP, subfasziale Lokalanästhetikainstillationen) angewendet werden sollten. Die in den letzten Jahren wieder aufgenommene Diskussion zu Vor- und Nachteilen der thorakalen Periduralanalgesie kann hier aus Platzgründen nicht differenziert dargestellt werden. Bei konventionellen kolorektalen Operationen ist die thorakale Periduralanalgesie unverändert die effektivste Schmerztherapie. Vorteile sind neben der optimalen Analgesie auch die Verkürzung der postoperativen Darmatonie. Nachteile sind Episoden postoperativer Hypotonie und Blasenentleerungsstörungen. Bei laparoskopischen Operationen im Rahmen eines Fast-track- oder ERAS-Programms ist der Wert der thorakalen Periduralanalgesie dagegen umstritten. Als Alternativen werden bei minimalinvasiver Chirurgie in jüngster Vergangenheit die Spinalanästhesie, intravenöses Lidocain und regionale Blockaden im Bereich der Bauchwand (TAP, kontinuierliche subfasziale LA-Instillation) diskutiert.

Minimalinvasive Operationstechnik: Laparoskopische kolorektale Resektionen gehen mit signifikanten und klinisch relevanten Vorteilen im postoperativen Verlauf einher (weniger Schmerzen, bessere Lungenfunktion, geringere Dauer der postopera- 
tiven Darmatonie und kürzere postoperative Verweildauer). Ob robotische Techniken mit weiteren Verbesserungen einhergehen, ist derzeit noch unklar. Wenn möglich, sollte die MIC im Rahmen von Fast-track oder ERAS Konzepten angewendet werden.

Vermeidung perioperativer Hyper- und Hypovolämie: Früher wurden durch falsche pathophysiologische Vorstellungen („Dritter Raum“) perioperative hochvolumige Infusionskonzepte favorisiert. Inzwischen ist unumstritten, dass eine liberale intraoperative Infusionstherapie zu höheren Komplikationsraten führt. Ob die sogenannte zielgerichtete Volumentherapie (,goal directed fluid therapy“ - GDFT) oder Nahe-Null-Volumenbilanzen (,near-zero fluid balance“ - NZFB) identische Ergebnisse erzielen oder GDFT bei Hoch-Risiko-Patienten doch vorteilhaft sein kann, ist umstritten. Infusionskonzepte, die mit einer perioperativen Gewichtszunahme von mehr als 2,5 kg einhergehen, müssen vermieden werden, da diese die Komplikationsraten erhöhen und die Genesung der Patienten beeinträchtigen.

Verzicht auf Sonden und Drainagen: Die Anwendung von intraperitonealen Drainagen führt nicht zu einer Reduktion der Häufigkeit oder einer früheren Erkennung von Anastomoseninsuffizienzen nach elektiven kolorektalen Resektionen und auch pelvine Drainagen haben nach tiefen anterioren Rektumresektionen keinen nachweisbaren Vorteil für den Patienten. Drainagen verunsichern andererseits die Patienten und erschweren die postoperative Mobilisation. Daher sollten im Rahmen optimierter perioperativer Behandlungskonzepte bei elektiven kolorektalen Resektionen keine intraperitonealen oder pelvinen Drainagen verwendet werden. Nasogastrale Sonden führen nach elektiven kolorektalen Operationen zu einer höheren Inzidenz von Pharyngitis, Laryngitis und pulmonalen Infekten. Nach abdominalchirurgischen Operationen ist die gastrointestinale Atoniedauer mit nasogastraler Sonde länger und die Pneumonierate höher als ohne Magensonde. Daher sollte auf Magensonden verzichtet werden oder sie sollten unbedingt am Ende der Operation entfernt werden.

\section{Weitere Elemente der optimalen Behandlung im Operationssaal}

Perioperative Antibiotikaprophylaxe: Die perioperative intravenöse „Single-Shot“-Antibiotikagabe (z. B. mit einem Cephalosporin und Metronidazol) innerhalb von 3060 Minuten vor der Hautinzision reduziert die postoperative Infektionsraten im Operationsgebiet von 39\% auf 13\%. Sie ist daher zurecht ein Bestandteil des optimalen perioperativen Managements.

Intraoperative Aufrechterhaltung der Körpertemperatur: Die negativen Folgen einer nicht beabsichtigen Hypothermie während abdominalchirurgischer Operationen sind gut dokumentiert. Eine Senkung der Körperkerntemperatur um $1^{\circ} \mathrm{C}$ erhöht die postoperative Komplikationsrate um etwa $10 \%$. Die intraoperative Aufrechterhaltung der Normothermie gelingt am besten durch aktive Zufuhr angewärmter Luft.

Standardisierte Narkose: Es gibt keine Hinweise dafür, dass eine total intravenöse Anästhesie (TIVA) gegenüber der Anwendung kurzwirksamer moderner Inhalationsanästhetika relevante Vorteile für den Patienten hat. Insgesamt scheint aber ein stan- 
dardisiertes Vorgehen bei der Narkoseführung unter Anwendung kurz-wirksamer Anästhetika, Narkosetiefe- und Relaxations-Monitoring unter Beachtung der Infusionsmenge (s. o.) im Rahmen der Fast-track- oder ERAS-Behandlung empfehlenswert zu sein.

\subsubsection{Optimales postoperatives Management}

\section{Kernelemente der postoperativen Behandlung}

Frühzeitige orale Ernährung: Die frühzeitige postoperative orale oder enterale Ernährung geht mit einer kürzeren Krankenhausverweildauer bei gleicher Komplikationsrate einher. Daher sollte die postoperative orale Ernährung bereits am Operationstag wieder aufgenommen werde.

Forcierte, frühe postoperative Mobilisation: Während die negativen Folgen anhaltender Bettruhe (thromboembolische Komplikationen, nachlassende Muskelkraft, größere postoperative Insulinresistenz) gut untersucht sind , sind hochwertige Publikationen zu den Effekten der frühen und forcierten postoperativen Mobilisation selten. Gleichwohl wird von allen Protagonisten der Fast-track oder ERAS -Behandlung die frühzeitige Mobilisation als Schlüssel zum Erfolg angesehen - auch wenn gleichzeitig eingestanden wird, dass dieses Element der multimodalen Therapie im klinischen Alltag am Schwierigsten zu realisieren ist.

\section{Weitere Elemente der postoperativen Behandlung}

Medikamentöse Thromboseprophylaxe: Die medikamentöse Thromboseprophylaxe ist unumstrittener Bestandteil einer optimalen perioperativen Behandlung bei elektiven kolorektalen Resektionen und als solche auch in einer S3-Leitlinie eindeutig beschrieben.

Postoperative Infusionstherapie: Die intravenöse Gabe von Flüssigkeit ist bei den meisten Patienten nach elektiven kolorektalen Resektionen nicht erforderlich, erfolgt aber aus traditionellen Gründen dennoch regelhaft. Im Gegensatz dazu sollte die postoperative Infusionsbehandlung mit Ende der Operation ebenfalls beendet werden. Patienten sollten spätestens 4 Stunden nach der Operation angehalten werden, klare Flüssigkeiten zu sich zu nehmen. Wenn postoperative Infusionen erforderlich werden (z. B. bei starker Übelkeit und/oder Erbrechen) sollten balancierte Elektrolytlösungen verabreicht werden. 0,9\%ige $\mathrm{NaCl}$-Lösung sollte wegen der bekannten Nebenwirkungen (hypochlorämische Azidose, Nierenfunktionsstörungen) als Standardinfusionslösung nicht mehr verwendet werden. Eine postoperative Oligurie $(<0,5 \mathrm{ml} / \mathrm{kg} / \mathrm{h}$ oder $<500 \mathrm{ml} / \mathrm{Tag})$ ist kein sicheres Hypovolämiezeichen und sollte nur zur Infusionsbehandlung führen, wenn zusätzliche Symptome der Hypovolämie (Tachykardie, Hypotonie, Schweißneigung, Verwirrtheit, verzögerte Kapillarfüllung) auftreten. 
Verzicht oder frühe Entfernung von Blasenkathetern: Die Verweildauer von Blasenkathetern ist mit der Häufigkeit von Harnwegsinfekten assoziiert, zudem behindern Blasenkatheter die postoperative forcierte Mobilisation der Patienten. Aus diesen Gründen sollten Blasenkatheter so rasch wie möglich nach der Operation entfernt werden.

Prophylaxe der postoperativen Darmatonie: Die postoperative Darmatonie (,postoperativer Ileus“) ist eine der wesentlichen Ursachen für Patientenbeschwerden. Sie führt zu verlangsamter Erholung und verlängert die Rekonvaleszenz- und Krankenhausdauer für Patienten mit elektiven abdominalchirurgischen Operationen. Daher sollen viele Elemente der Fast-track-Rehabilitation dabei helfen, die postoperative gastrointestinale Atonie zu vermeiden: Reduktion der Opioiddosis durch regionale Analgesietechniken, minimalinvasive Chirurgie, Verzicht auf die Magensonde, geringe i.v. Flüssigkeits-/Natrium-Zufuhr, rasche orale Ernährung, forcierte Mobilisation. In mehreren randomisierten kontrollierten Studien hat das Kauen von Kaugummi einen positiven Effekt auf die postoperative Darmmotilität und die Atoniedauer. Orale Laxantien reduzieren die Dauer bis zum ersten Stuhlgang, beeinflussen aber eine obere gastrointestinale Atonie nicht.

\subsubsection{Organisation des optimalen perioperativen Managements}

Die konsequente Umsetzung der einzelnen Elemente der Fast-track oder ERAS-Behandlung ist in zahlreichen Studien mit einem besseren Ergebnis für die behandelten Patienten assoziiert. Die Gesamtkomplikationsrate, die Rate infektiöser Komplikationen, die Dauer des stationären Aufenthaltes und die Wiederaufnahmeraten sind umso besser, je mehr Elemente des multimodalen Behandlungskonzeptes angewendet wurden. Erfolg oder Misserfolg von Fast-track und ERAS sind also streng mit der Compliance in der Durchführung der einzelnen Elemente verbunden.

Die Einführung des optimierten, multimodalen perioperativen Managements verändert die Behandlungsroutinen der traditionellen perioperativen Behandlung in erheblichem Maße. Zahlreiche Handlungsweisen aus der bisherigen klinischen Routine werden verändert, so dass das gesamte Behandlungsteam bezüglich seiner Gewohnheiten verunsichert wird. Gleichzeitig sind die Veränderungen so umfassend, dass weder Ärzte noch Pflegende die Umstellungen ohne weiteres bewältigen und das Fast-track-Management bei jedem Patienten konsequent umsetzen können. Sowohl im Ausland als auch in der eigenen Klinik hat sich daher die Etablierung sogenannter Fast-track-Assistenten oder ERAS-Schwestern/Pfleger bewährt. Fast-trackAssistentinnen koordinieren die gemeinsamen Anstrengungen aller Berufsgruppen und kontrollieren gleichzeitig die vollständige Umsetzung aller Fast-track-Elemente und Maßnahmen. Fast-track-Assistentinnen sind für die konsequente Umsetzung aller Fast-track-Elemente außerhalb des Operationssaals zuständig. Fast-track-Assistentinnen schulen, informieren und motivieren ihre Patienten während des gesamten 
Behandlungsverlaufs, so dass die Beziehung Fast-track-Assistentin zu Patient eher der eines Trainers zum Athleten als der eines traditionellen Pflegenden zum Patienten entspricht. Gleichzeitig verbessern Fast-track-Assistentinnen die Kommunikation sowohl zwischen Patienten und medizinischem Fachpersonal als auch innerhalb der medizinischen Berufsgruppen.

Die gemeinsame Festlegung von Rekonvaleszenz- und Entlassungskriterien durch Chirurg, Fast-track-Assistentin und Patient bereits vor der stationären Aufnahme, sind bei der Etablierung des optimalen perioperativen Managements von besonderer Bedeutung. Nur nach Vorgabe eines Entlassungsziels (z. B. Entlassung am 5. Tag nach elektiver Kolonresektion) und gleichzeitiger Festlegung von Etappenzielen (z. B. Mobilisation aus dem Bett am Operationstag und Essen und Trinken ab dem 1. postoperativen Tag) können Patienten vollständig an einer raschen Genesung nach einer Operation mitarbeiten. Raschere Erholung, besseres Befinden, größere Mobilität, frühzeitige Autonomie und Homöostase sind die entscheidenden Ziele des Fasttrack. Die früher als zuvor übliche Entlassung aus dem Krankenhaus bei gelungenem perioperativem Management ist dann nur die erwünschte Nebenwirkung!

\section{Weiterführende Literatur}

Allen C, Glasziou P, Del Mar C. Bed rest: a potentially harmful treatment needing more careful evaluation. Lancet. 1999;354(9186):1229-1233.

Bardram L, Funch Jensen P, Jensen P, Crawford ME, Kehlet H. Recovery after laparoscopic colonic surgery with epidural analgesia, and early oral nutrition and mobilisation. Lancet. 1995;345(8952):763-764.

Brandstrup B, Svendsen PE, Rasmussen M, et al. Which goal for fluid therapy during colorectal surgery is followed by the best outcome: near-maximal stroke volume or zero fluid balance? British journal of anaesthesia. 2012;109(2):191-199.

Brandstrup B, Tonnesen H, Beier-Holgersen R, et al. Effects of intravenous fluid restriction on postoperative complications: comparison of two perioperative fluid regimens: a randomized assessor-blinded multicenter trial. Annals of surgery. 2004;240(2):386-388.

Bretagnol F, Panis Y, Rullier E, et al. Rectal cancer surgery with or without bowel preparation: The French GRECCAR III multicenter single-blinded randomized trial. Annals of surgery. 2010;252(5):863-868.

Denost Q, Rouanet P, Faucheron JL, et al. To Drain or Not to Drain Infraperitoneal Anastomosis After Rectal Excision for Cancer: The GRECCAR 5 Randomized Trial. Annals of surgery. 2017;265(3):474-480.

DREAMS Trial Collaborators and West Midlands Research Collaborative. Dexamethasone versus standard treatment for postoperative nausea and vomiting in gastrointestinal surgery: randomised controlled trial (DREAMS Trial). BMJ. 2017;357:j1455.

Gustafsson UO, Scott MJ, Hubner M, et al. Guidelines for Perioperative Care in Elective Colorectal Surgery: Enhanced Recovery After Surgery $\left(\right.$ ERAS $\left.^{\circledR}\right)$ Society Recommendations: 2018. World journal of surgery. 2019;43(3):659-695.

Heaton KW. Dangers of bed rest. Lancet. 1999;354(9194):2004.

Holte K, Nielsen KG, Madsen JL, Kehlet H. Physiologic effects of bowel preparation. DisColon Rectum. 2004;47(8):1397-1402. 
Kaufner C. S3-Leitlinie Präoperative Anämie AWMF online. Das Portal der wissenschaftlichen Medizin 2018. https://www.awmf.org/uploads/tx_szleitlinien/001-0241_S3_PraeoperativeAnaemie_2018-04.pdf.

Koullouros M, Khan N, Aly EH. The role of oral antibiotics prophylaxis in prevention of surgical site infection in colorectal surgery. International journal of colorectal disease. 2017;32(1):1-18.

McSorley ST, Steele CW, McMahon AJ. Meta-analysis of oral antibiotics, in combination with preoperative intravenous antibiotics and mechanical bowel preparation the day before surgery, compared with intravenous antibiotics and mechanical bowel preparation alone to reduce surgical-site infections in elective colorectal surgery. BJS Open. 2018;2(4):185-194.

Mei B, Wang W, Cui F, Wen Z, Shen M. Chewing Gum for Intestinal Function Recovery after Colorectal Cancer Surgery: A Systematic Review and Meta-Analysis. Gastroenterol Res Pract. 2017;2017:3087904.

Rollins KE, Javanmard-Emamghissi H, Lobo DN. Impact of mechanical bowel preparation in elective colorectal surgery: A meta-analysis. World journal of gastroenterology : WJG. 2018;24(4):519-536.

Schwenk W, Haase 0, Neudecker J, Muller JM. Short term benefits for laparoscopic colorectal resection. Cochrane database of systematic reviews. 2005(3):CD003145.

Watson DJ. Nurse coordinators and ERAS programs. Nurs Manage. 2018;49(1):42-49.

Weimann A, Breitenstein S, Breuer JP, et al. Klinische Ernährung in der Chirurgie. S3-Leitlinie der Deutschen Gesellschaft fur Ernährungsmedizin e. V. Der Chirurg; Zeitschrift fur alle Gebiete der operativen Medizen. 2014;85(4):320-326.

Zhang HY, Zhao CL, Xie J, et al. To drain or not to drain in colorectal anastomosis: a meta-analysis. International journal of colorectal disease. 2016;31(5):951-960.

Zingg U, Miskovic D, Pasternak I, et al. Effect of bisacodyl on postoperative bowel motility in elective colorectal surgery: a prospective, randomized trial. International journal of colorectal disease. 2008;23(12):1175-1183.

Zwissler B. Präoperative Evaluation erwachsener Patienten vor elektiven, nicht Herz-Thorax-chirurgischen Eingriffen: Gemeinsame Empfehlung der Deutschen Gesellschaft für Anästhesiologie und Intensivmedizin, der Deutschen Gesellschaft fur Chirurgie und der Deutschen Gesellschaft für Innere Medizin. Der Anaesthesist. 2017;66(6):442-458. 
